\title{
Magnetic field dependence of energy levels in biased bilayer graphene quantum dots
}

\author{
D. R. da Costa, ${ }^{1,2, *}$ M. Zarenia, ${ }^{2, \dagger}$ Andrey Chaves,,${ }^{1,3}$ G. A. Farias, ${ }^{1}$ and F. M. Peeters ${ }^{2,1, \ddagger}$ \\ ${ }^{1}$ Departamento de Física, Universidade Federal do Ceará, 60455-900 Fortaleza, Ceará, Brazil \\ ${ }^{2}$ Department of Physics, University of Antwerp, Groenenborgerlaan 171, B-2020 Antwerp, Belgium \\ ${ }^{3}$ Department of Chemistry, Columbia University, 3000 Broadway, New York, New York 10027, USA \\ (Received 25 November 2015; revised manuscript received 19 January 2016; published 1 February 2016)
}

\begin{abstract}
Using the tight-binding approach, we study the influence of a perpendicular magnetic field on the energy levels of hexagonal, triangular, and circular bilayer graphene (BLG) quantum dots (QDs) with zigzag and armchair edges. We obtain the energy levels for $A B$ (Bernal)-stacked BLG QDs in both the absence and the presence of a perpendicular electric field (i.e., biased BLG QDs). We find different regions in the spectrum of biased QDs with respect to the crossing point between the lowest-electron and -hole Landau levels of a biased BLG sheet. Those different regions correspond to electron states that are localized at the center, edge, or corner of the BLG QD. Quantum Hall corner states are found to be absent in circular BLG QDs. The spatial symmetry of the carrier density distribution is related to the symmetry of the confinement potential, the position of zigzag edges, and the presence or absence of interlayer inversion symmetry.
\end{abstract}

DOI: 10.1103/PhysRevB.93.085401

\section{INTRODUCTION}

Recent progress in the fabrication and characterization of graphene-based nanodevices [1-3] has made possible the study of electronic, optical, and transport properties of different graphene-based nanostructures [4,5]. In this context, graphene quantum dots (QDs) have been identified as attractive candidates for spin qubits and quantum information storage [6-8]. It has been known that lateral confinement of Dirac fermions using an electrostatic gate potential is a challenging task due to the Klein tunneling effect, which prevents electrical confinement of carriers in graphene [9]. Instead, graphene QDs can be realized by cutting small flakes from a graphene sheet [10]. It was shown that the energy states of such QDs are strongly dependent on the shape, size, and type of the boundary edges [11-17]. On the other hand, it has been shown that bound electron or hole states can be achieved for the special case of zero-energy modes [18,19].

In contrast to monolayer graphene, an electrostatic potential difference can be introduced in bilayer graphene (BLG) by chemical doping [20] or by the application of a perpendicular electric field [21-23]. This leads to an inversion asymmetry between the layers and opens an energy gap in the energy spectrum. Tailoring the gap, by using applied gate potentials, leads to the creation of electrostatic confined QDs in BLG, as has been experimentally [21,24,25] and theoretically [26-34] reported in the literature. The energy spectra of such QDs are not determined by the type of edges or the disorder present at the edges. However, experimental works demonstrated the realization of controllable gates to tune the electronic band gap up to about $250 \mathrm{meV}$ in BLG [35], which limits the barrier height for electrostatic confinement in BLG quantum dots. Therefore, to realize larger quantum confinement, one must rely on finite-size systems, where the type of edge crucially determines the character of the low-energy electronic

\footnotetext{
*diego_rabelo@fisica.ufc.br

†mohammad.zarenia@uantwerpen.be

${ }^{\ddagger}$ francois.peeters@uantwerpen.be
}

states. For instance, graphene QDs with zigzag edges exhibit a degenerate zero-energy state [11-17] not found in the well-known semiconductor-based QDs.

In our recent paper [34], we showed that the edges of BLG QDs also play an important role in their electronic properties, as do the type of stacking of the graphene layers. Those results were obtained in the absence of a magnetic field. It has been shown that monolayer and bilayer graphene display new properties in the presence of a perpendicular magnetic field not found in typical semiconductors [15,27,28,30,36-40]. This motivated us to extend our previous study [34] and to investigate the influence of a magnetic field on the energy levels of BLG QDs with triangular, hexagonal, and circular shapes (see Fig. 1), for different types of edges.

The present paper is organized as follows: In Sec. II, the tight-binding concepts and the assumed settings used in our numerical calculations are briefly discussed. In Secs. III and IV, the energy spectra for the Bernal-stacked BLG QDs with armchair and zigzag edges are discussed, respectively. In Sec. V, we present our results for circular BLG QDs, in which the QD boundary is a combination of both armchair and zigzag edges. Our final remarks are given in Sec. VI.

\section{NUMERICAL METHOD}

Bilayer graphene consists of two coupled graphene layers linked by the van der Waals interaction [41]. Carriers in BLG are described by the following tight-binding Hamiltonian:

$$
H=\sum_{i \neq j}\left(\tau_{i j} c_{i}^{\dagger} c_{j}+\text { H.c. }\right)+\sum_{i}\left(\epsilon_{i}+M_{i}+V_{i}\right) c_{i}^{\dagger} c_{i},
$$

where $c_{i}\left(c_{i}^{\dagger}\right)$ annihilates (creates) an electron in site $i$ with on-site energy $\epsilon_{i} . \tau_{i j}$ is the nearest-neighbor hopping parameter, which for intralayer hopping contributions are $\tau_{i j}=t=-2.7 \mathrm{eV}$, whereas the interlayer ones are $\tau_{i j}=t_{\perp}=$ $-0.4 \mathrm{eV}$. In an $A B$-stacked BLG, the atoms in the $A(B)$ sublattice in the bottom layer are linked with $B(A)$ atoms in the top layer. $M_{i}$ and $V_{i}$ are the on-site mass potential term and the on-site electrostatic potential, respectively, used to open a gap 


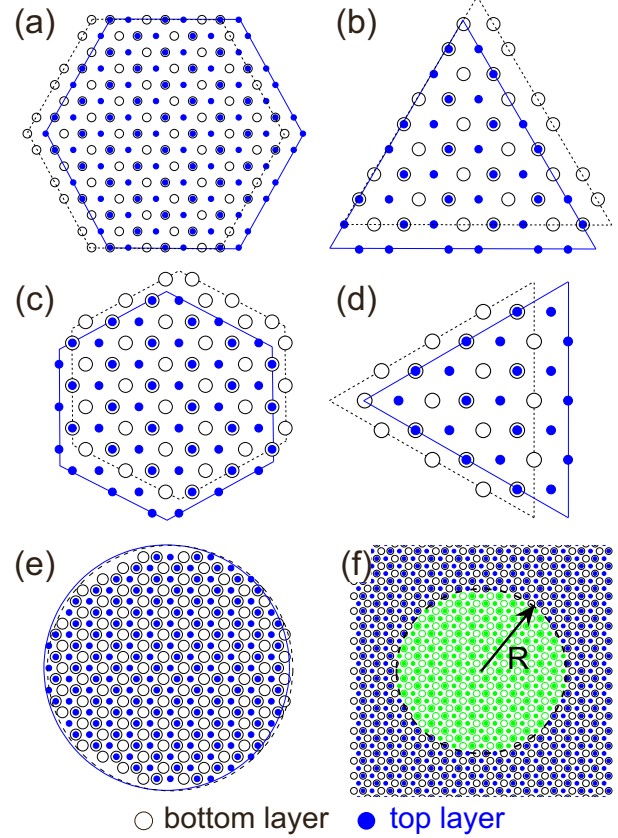

FIG. 1. Sketches of (a),(b) armchair and (c),(d) zigzag BLG QDs with (a),(c) hexagonal, (b),(d) triangular, and (e),(f) circular geometries considered in this work. The first four geometries are characterized by the number of carbon hexagons $N$ at their edges. The circular dots (e) and (f) are characterized by their radius $R$. (a)-(e) QDs are defined by cutting the BLG lattice and (f) is defined by a size-dependent staggered potential $M_{i}$, where the atoms belonging to sublattices $A_{b}\left(A_{u}\right)$ and $B_{b}\left(B_{u}\right)$ have a mass-term potential given by $+M_{0}\left(-M_{0}\right)$ and $-M_{0}\left(+M_{0}\right)$, respectively, and $M_{i}=0$ in the region inside the dot, represented in green. Bottom (top) atoms are given by black open (blue closed) symbols.

in the energy spectrum of BLG and, consequently, to simulate electronic confinement in BLG nanostructures $[32,33]$. The staggered site-dependent potential is defined in such a way that $M_{i}=M_{0}\left(M_{i}=-M_{0}\right)$ if $i$ belongs to the lattice $A(B)$ in one layer and $B(A)$ in the other one [33]. This breaks the sublattice symmetry in BLG QDs. Similar to monolayer graphene [42-44], depositing BLG on the appropriate substrate, e.g., $\mathrm{SiC}$ and hexagonal boron nitride, may induce such mass-term potential. For the case of biased BLG QDs, we take $V_{i}=V$ for the atoms in the upper layer and $V_{i}=-V$ for the atoms in the lower layer. The effect of an external magnetic field is incorporated in the tight-binding model via the Peierls substitution, as $\tau_{i j} \rightarrow \tau_{i j} \exp \left[i \frac{e}{\hbar} \int_{j}^{i} \vec{A} \cdot d \vec{l}\right]$, where $\vec{A}=(0, B x, 0)$ is the vector potential in the Landau gauge, giving $\vec{B}=B \hat{z}$ as an external magnetic field perpendicular to the BLG flakes. For the chosen gauge, one finds that the Peierls phase is only nonzero in the $y$ direction and is given by $\exp \left[i \frac{2 \pi x}{3 a} \frac{\phi}{\phi_{0}}\right]$, where $a=0.142 \mathrm{~nm}$ is the lattice parameter of graphene, $\phi_{0}=h / e$ is the magnetic quantum flux, and $\phi=3 \sqrt{3} a^{2} B / 2$ is the magnetic flux through a carbon hexagon. In order to facilitate the comparison, our results for the different geometries and boundaries are obtained for BLG QDs with the same confinement area, $S \approx(16.7)^{2} \mathrm{~nm}^{2}$, such that the considered hexagonal and triangular BLG QDs with

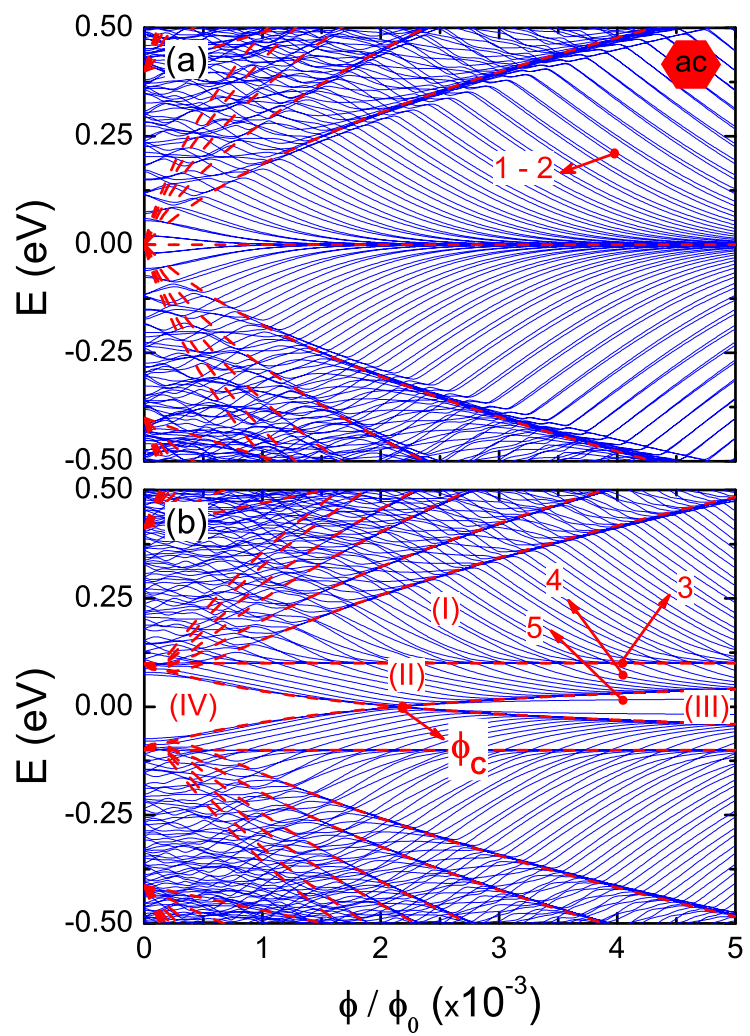

FIG. 2. Energy levels (a) in the absence $(V=0)$ and (b) in the presence $(V=0.1 \mathrm{eV})$ of a bias potential as a function of the magnetic flux $\left(\phi / \phi_{0}\right)$ through a hexagonal BLG QD with armchair edges and side length $L \approx 10.366 \mathrm{~nm}(N=25$ carbon hexagons in each side), as sketched in Fig. 1(a). The red dashed curves are the LLs of an infinite BLG sheet.

armchair and zigzag edges have the side length $L^{H} \approx 10 \mathrm{~nm}$ and $L^{T} \approx 25 \mathrm{~nm}$, respectively.

\section{ARMCHAIR EDGES}

First, we consider BLG QDs with armchair edges, for which it is known that edge states are absent. We will discuss the similarities and differences between the energy spectra obtained for hexagonal and triangular geometries in the absence and presence of an electrostatic bias potential.

Figure 2 shows the energy levels of an armchair hexagonal BLG QD with side length $L \approx 10 \mathrm{~nm}$ (which corresponds to $N=25$ carbon hexagons in each length) as a function of magnetic flux threading one carbon hexagon $\phi$, respectively, for (a) $V=0$ and (b) $V=0.1 \mathrm{eV}$. Just like in monolayer graphene QDs [11,12], the BLG QD spectra are always composed of a large set of energy states, so that Landau levels (LLs) of an infinite BLG sheet, analytically calculated within the continuum approximation, match the regions of the QD spectrum with higher density of states. For lower magnetic fields, the LLs of the bulk system are not meaningful due to the important role played by the finite size of the system, especially for higher-energy states, where in addition band warping also perturbs the energy levels obtained by the continuum approximation. However, as the magnetic field increases, the 


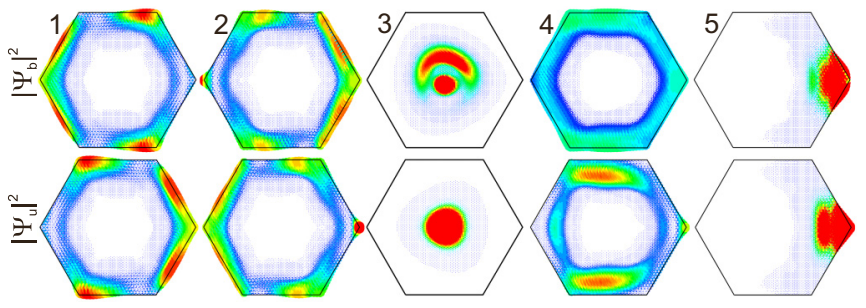

FIG. 3. The probability density for the bottom $\left|\Psi_{b}\right|^{2}$ and top $\left|\Psi_{u}\right|^{2}$ graphene layers is shown for states labeled in Fig. 2 by the points $1(E \approx 0.20182 \mathrm{eV}), 2(E \approx 0.21093 \mathrm{eV}), 3(E \approx 0.1 \mathrm{eV}), 4(E \approx$ $0.07421 \mathrm{eV})$, and $5(E \approx 0.01587 \mathrm{eV})$, respectively, for the unbiased and biased cases and for $\phi / \phi_{0}=4.0 \times 10^{-3}$. Red (blue) colors stand for high (low) density.

magnetic length becomes smaller than the system size, so that confinement effects are strongly reduced, and the magnetic levels in the BLG QDs, i.e., the so-called Fock-Darwin levels, converge to the analytical LLs of an infinite BLG sheet (shown by the dashed red curves in both spectra). The LL spectrum $E^{(n)}$ of a symmetrically biased BLG (with applied bias potential $V$ ) is determined by $[27,28]$

$$
\begin{aligned}
& \left.\left[\left(E^{(n)}+V\right)^{2}-2(n+1) \gamma^{2}\right)\right]\left[\left(E^{(n)}-V\right)^{2}-2 n \gamma^{2}\right] \\
& \quad-\left(E^{(n)^{2}}-V^{2}\right) t_{\perp}^{2}=0,
\end{aligned}
$$

where $n$ is an integer and $\gamma=\hbar v_{F} / l_{B}$, with $v_{F} \approx 10^{6} \mathrm{~m} / \mathrm{s}$ the Fermi velocity and $l_{B}=\sqrt{\hbar / e B}$ the magnetic length. The magnetic field is related to the magnetic flux through the QDs by $B=(4 \pi \hbar \phi) /\left(3 \sqrt{3} e a^{2} \phi_{0}\right)$. Equation (2) gives eight solutions for each $n$. The four lowest LLs start at $E= \pm V$ and the four highest LLs start at $E= \pm t_{\perp}[27,28]$. In the absence of bias $V=0$ [Fig. 2(a)] as the magnetic flux increases, the energy levels approach the zeroth LL (i.e., $E=0$ ) in pairs. Notice that these pairs of states have very close energies but are nondegenerate and are the so-called quantum Hall edge states. The corresponding electron densities for two of these states [see labels 1 and 2 in Fig. 2(a)] are shown in Fig. 3. The electron density for the bottom $\left|\Psi_{b}\right|^{2}$ and upper $\left|\Psi_{u}\right|^{2}$ layers is shown separately. Due to the layer symmetry in BLG QDs when $V=0$, the wave functions in each layer can be (nearly) transformed into each other by a $\pi$ rotation and thus the total probability density for states labeled by 1 and 2 has the same kind of symmetry and shape. For the nonzero bias case [Fig. 2(b)], an energy gap opens around $E=0$ and the zeroth unbiased LL is now broken into four levels. For $n=0$, the lowest four solutions of Eq. (2) are $E^{(0)}= \pm V$ and [28]

$$
E_{ \pm}^{(0)}= \pm V \frac{\left(t_{\perp}^{2}-2 \beta \hbar^{2} v_{F}^{2}\right)}{\left(t_{\perp}^{2}+2 \beta \hbar^{2} v_{F}^{2}\right)},
$$

with $\beta=(4 \pi \phi) /\left(3 \sqrt{3} a^{2} \phi_{0}\right)$. Equation (3) is obtained by assuming $E, V \ll t_{\perp}$. It is worthwhile to emphasize that one by one, those pairs of states pass through $E= \pm V$ and, while one of them approaches the zeroth biased LLs $\left(E_{ \pm}^{(0)}\right)$, the other one approaches $E= \pm V . E_{ \pm}^{(0)}$ cross each other at

$$
\phi_{C}=\frac{3 \sqrt{3} a^{2} \phi_{0}}{8 \pi \hbar^{2} v_{F}^{2}}\left(V^{2}+t_{\perp}^{2}\right) .
$$

Similar to the unbiased case, given by Fig. 2(a), the energy levels in the presence of a bias potential $V=0.1 \mathrm{eV}$ exhibit electron-hole symmetry $E_{e}=-E_{h}$ and the twofold degeneracy present at $\phi / \phi_{0}=0$ is broken for $\phi / \phi_{0} \neq 0$. The energy levels in the spectrum of Fig. 2(b) can be separated into the following four regions (which are indicated in the figure):

(i) $V<|E|<E^{(1)}$, which corresponds to pairs of quantum Hall edge states that decrease in energy with increasing magnetic flux in pairs. The corresponding wave functions of those states are similar to those shown in panels 1 and 2 of Fig. 3. For energies $E \approx \pm V$, we expect to observe centered-peak localized states corresponding to the zeroth-LL wave function (see panel 3 of Fig. 3).

(ii) $|E|<V$ and $E>E_{+}^{(0)}$ for $\phi<\phi_{C}$ and $E>E_{-}^{(0)}$ for $\phi>\phi_{C}$ : Due to the breaking of the layer symmetry in this region, the symmetry of the paired quantum Hall edge states in region (i) is broken and one of the paired states approaches the biased potential $V$ and the other enters into region (ii) and approach the zeroth LLs $E_{ \pm}^{(0)}$. The corresponding layer electron densities $\left|\Psi_{u}\right|^{2}$ and $\left|\Psi_{b}\right|^{2}$ are shown in Fig. 3 for one of these states, labeled by 4 in Fig. 2(b). The distribution of electrons in each layer is different and there is no layer symmetry.

(iii) $E<E_{-}^{(0)}$ and $E>E_{+}^{(0)}$ and $\phi>\phi_{C}$ : We observe states in this region which exhibit slightly different behaviors than those quantum Hall edge states in regions (i) and (ii). These states are confined at the corners of the QD (see panel 5 in Fig. 3) and occur in the energy gap between the electron and hole levels of biased infinite BLG. We will analyze and discuss these remarkable corner states further in the paper.

(iv) $E>E_{-}^{(0)}$ and $E<E_{+}^{(0)}$ and $\phi<\phi_{C}$ : In this region, the spectrum of armchair hexagonal BLG QDs displays an energy gap between the electron and hole states. We will see later that in the presence of zigzag edges, edge states due to the zigzag boundaries will appear in this region.

The energy spectrum of Fig. 4 for the armchair triangular QDs is similar to that of the armchair hexagonal QDs shown in Fig. 2. The similarity comes from the fact that both armchair triangular and hexagonal BLG QDs have similar edge structures [see Figs. 1(a) and 1(b)]. In Fig. 4(a), the quantum Hall edge states approaching the zeroth LLs are almost twofold degenerate [see Fig. 4(c), which shows an enlargement for low energies]. The states labeled by points 1 and 2 have very close energies $\left(E_{1}=0.0345\right.$ and $\left.E_{2}=0.0368 \mathrm{eV}\right)$ and their wave functions are almost layer symmetric [Fig. 4(d)], such that the electron densities for the upper and lower layers can be transformed into each other by a reflection with respect to the black dashed line.

In order to understand the states in region $E_{+}^{(0)}<E<E_{-}^{(0)}$ and $\phi>\phi_{C}$ of the biased energy spectrum shown in Fig. 4(b), we depict in Fig. 5(b) the contributions of the different layers as well as the total electron density for the states labeled 1-7 in Fig. 5(a). Notice that at $\phi=\phi_{C}$, the spectrum exhibits an anticrossing between the electron and hole states. At this point, the electron densities exhibit a centered-peak state, i.e., characterizing the zeroth-LL state [see panels 1 and 2 in Fig. 5(b)]. Notice that the corresponding layer densities can be transformed into each other by $\left|\Psi_{b}^{e, h}\right|^{2}=\left|\Psi_{u}^{h, e}\right|^{2}$ due to the electron-hole symmetry of the energy spectrum. With increasing magnetic field $\phi>\phi_{C}$, the electron distributions 


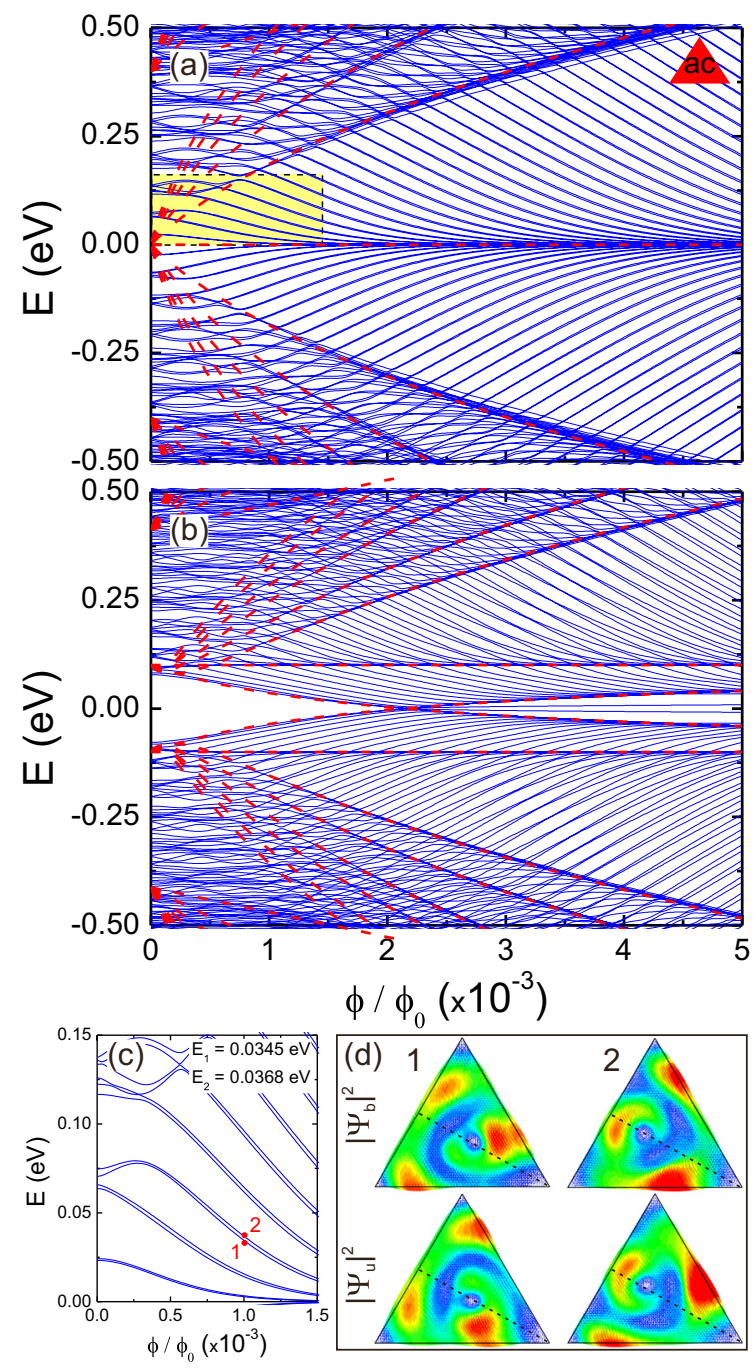

FIG. 4. (a),(b) The same as in Figs. 2(a) and 2(b), but now for the armchair triangular BLG QD with lateral size $L \approx 25.418 \mathrm{~nm}$ (equivalent to $N=60$ carbon hexagons in each side), as sketched in Fig. 1(b). The red dashed curves are the LLs of an infinite BLG sheet. (c) An enlargement of the spectrum for low energies. (d) The probability density for the bottom $\left|\Psi_{b}\right|^{2}$ and top $\left|\Psi_{u}\right|^{2}$ layers is shown for states 1 and 2 denoted in (c) with energies $E_{1}=0.0345$ and $E_{2}=0.0368 \mathrm{eV}$. Red (blue) colors stand for high (low) density.

move to the corner of the triangular dot (see the electron densities for the energy points 2-7). This feature is similar to those we observed in panel 5 of Fig. 3 for the hexagonal armchair QDs.

Figures 6(a) and 7(a) show a zoom of the energy spectrum around $|E| \leqslant V$, respectively, for the hexagonal and triangular BLG QDs with armchair edges. One can see that as the magnetic flux increases, more states appear in the region $E_{+}^{(0)} \lesssim E_{n} \lesssim E_{-}^{(0)}$ and $\phi>\phi_{C}$. Our results for the electron densities demonstrate that the two lowest-energy states are confined at the corners of the dot. The corresponding total probability densities of these states are shown in panels 1 and 2 in Figs. 6(b) and 7(b). Due to electron-hole symmetry, they can be transformed into each other by a phase rotation of $\pi$ for the hexagonal case and $2 \pi / 3$ for the triangular QD due
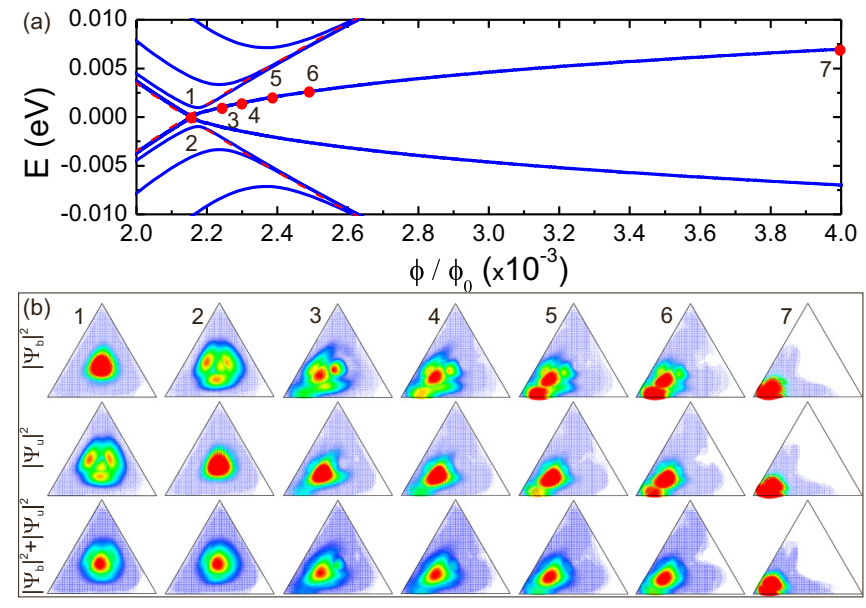

FIG. 5. (a) An enlargement of the energy spectrum around the crossing point $\phi_{C}$ for a biased triangular BLG QD [see Fig. 4(b)]. The red dashed curves are the two lowest LLs of an infinite sheet of BLG. (b) The probability density contribution from each layer (bottom $\left|\Psi_{b}\right|^{2}$ and top $\left.\left|\Psi_{u}\right|^{2}\right)$, as well as the total density $\left(\left|\Psi_{b}\right|^{2}+\left|\Psi_{u}\right|^{2}\right)$, are shown for states labeled by $1\left(E \approx 1.7039 \times 10^{-4} \mathrm{eV}\right), 2(E \approx-1.7039 \times$ $\left.10^{-4} \mathrm{eV}\right), 3\left(E \approx 9.9892 \times 10^{-4} \mathrm{eV}\right), 4\left(E \approx 1.3996 \times 10^{-3} \mathrm{eV}\right)$, $5\left(E \approx 2.0607 \times 10^{-3} \mathrm{eV}\right), 6\left(E \approx 2.6142 \times 10^{-3} \mathrm{eV}\right)$, and $7(E \approx$ $6.9856 \times 10^{-3} \mathrm{eV}$ ) in the energy spectrum. Red (blue) colors stand for high (low) density.

to the $C_{3}$ rotation symmetry. We call these states quantum Hall corner states. We will show in Sec. V that in the case of a circular dot (with no corners), these states are absent. As we go to higher-energy levels, the electrons (holes) distribute along the edges of the QD [see panels 3-8 in Figs. 6(b) and 7(b)]. In
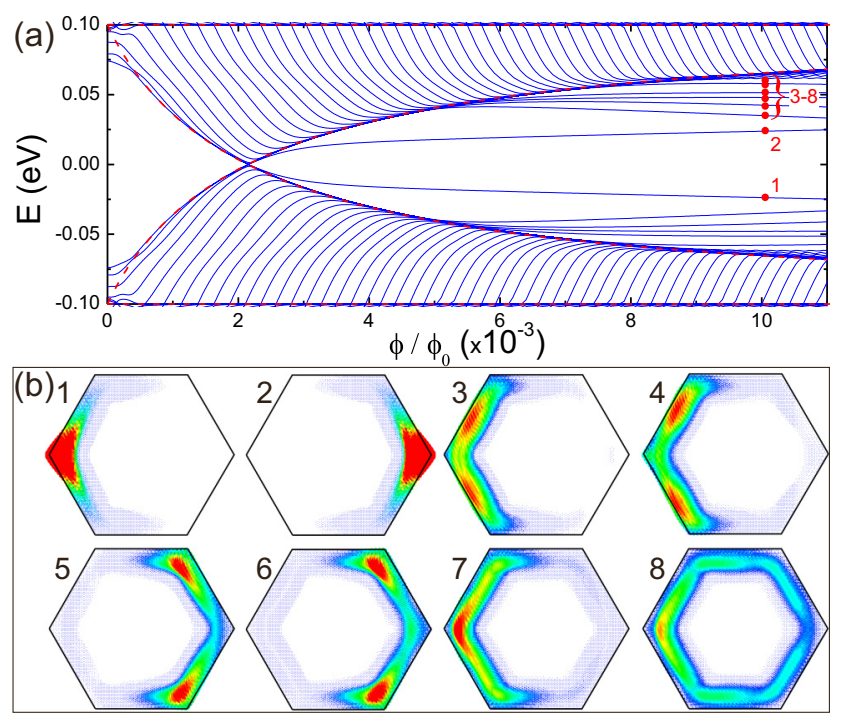

FIG. 6. (a) A zoom of the magnetic energy spectrum for a hexagonal armchair BLG QD [Fig. 2(b)] near the zero-energy level. The red dashed curves are the lowest zeroth LLs of an infinite sheet of BLG. (b) The total probability densities are shown for states labeled by 1 to 8 at $\phi / \phi_{0}=10.0 \times 10^{-3}$. Red (blue) colors stand for high (low) density. 

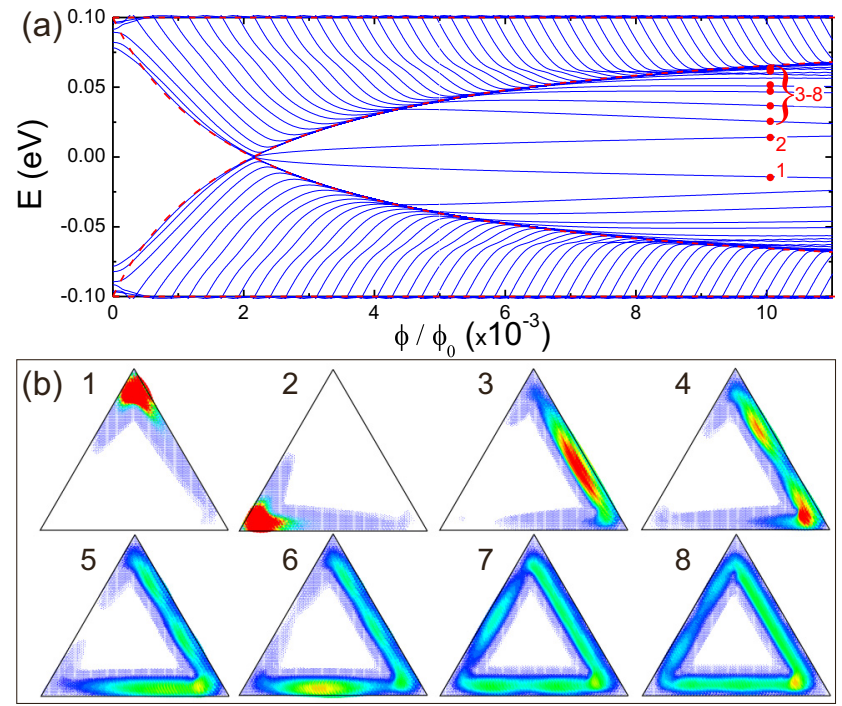

FIG. 7. The same as Fig. 6, but now for the armchair triangular BLG QD.

fact, these states approach the quantum Hall edge states in the regions $E_{-}^{(0)}<E<V$ and $-V<E<E_{+}^{(0)}$.

\section{ZIGZAG EDGES}

The energy spectra of hexagonal BLG QDs with zigzag edges and side length $L \approx 10 \mathrm{~nm}$, which corresponds to $N=41$ carbon hexagons at each side, are shown in Figs. 8(a) and 8 (b) as a function of magnetic flux, respectively, for zero and nonzero bias voltage. Due to the zigzag edges, the energy spectrum of the unbiased hexagonal BLG QD exhibits a highly degenerate zero-energy level. These zero-energy levels are edge states and therefore the corresponding spectrum exhibits a very distinct behavior as compared to the armchair BLG QDs discussed in Figs. 2 and 4. The spectrum in Fig. 8(a) is similar to the spectrum of the hexagonal monolayer graphene QDs with zigzag edges [14]. Notice that the zero-energy states of the zigzag hexagonal QD without bias potential are not affected by the magnetic field because they are strongly confined at the edges of the dot. By applying the bias potential [Fig. 8(b)], we observe that the zero-energy levels are broken into two bunches of levels with $E \approx+V$ and $E \approx-V$. This is indeed due to the breaking of the layer symmetry, which opens an energy gap in the spectrum. In contrast to armchair BLG QDs, zigzag-edge states appear in the region $|E|<V$ and $\phi<\phi_{C}$ (compare Fig. 8 with Figs. 2 and 4). These states exhibit an oscillatory behavior as a function of the magnetic flux, which is due to the interplay between the zigzag edges of both layers and the bias potential. In order to provide a better understanding of this oscillatory behavior of the states inside the $|E|<V$ region and for $\phi<\phi_{C}$, we show an enlargement around the low-energy levels in Fig. 9(a). Due to the interplay between the zigzag edges and the external magnetic field, anticrossings appear in the energy spectrum. An anticrossing point around $\phi / \phi_{0}=0.22 \times 10^{-3}$ is enlarged in the inset of Fig. 9(a). Figure 9(b) shows the corresponding total electron densities for the points labeled by 1 to 6 around the anticrossing between the ground states of the electron

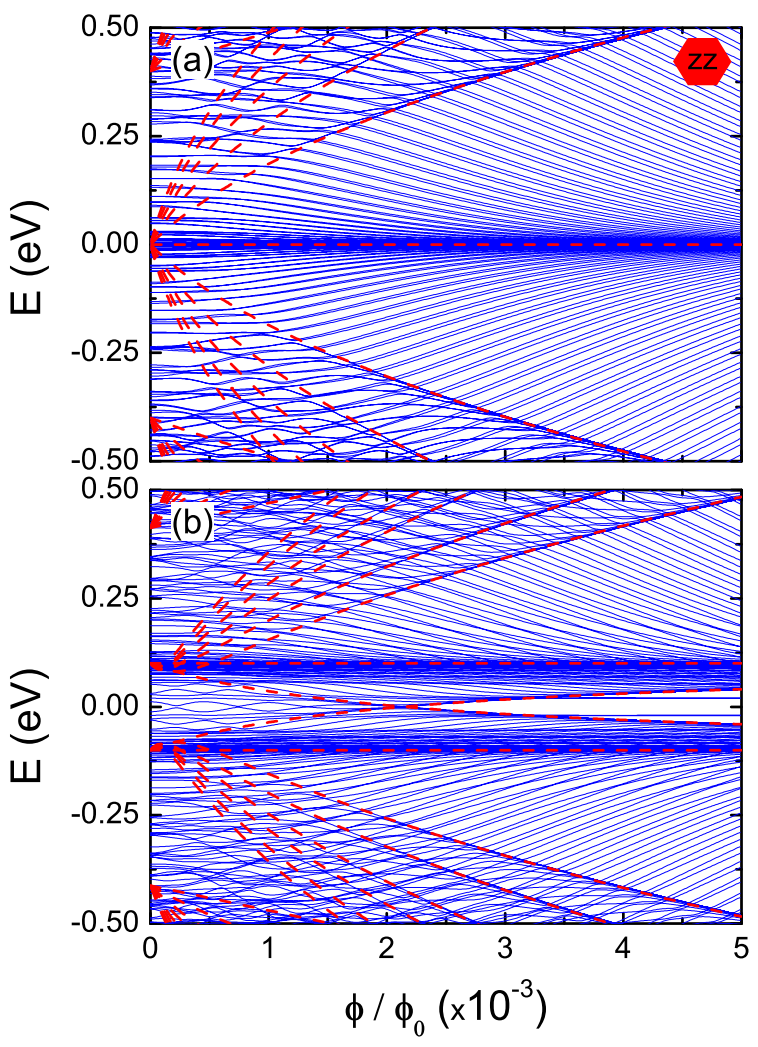

FIG. 8. Energy levels (a) in the absence and (b) in the presence of a bias potential as a function of the magnetic flux $\left(\phi / \phi_{0}\right)$ for a hexagonal BLG QD with zigzag edges and $L \approx 10.084 \mathrm{~nm}(N=41$ carbon hexagons in each side), as sketched in Fig. 1(c). The red dashed curves are the LLs of an infinite sheet of BLG. The bias potential is $V=0.1 \mathrm{eV}$.
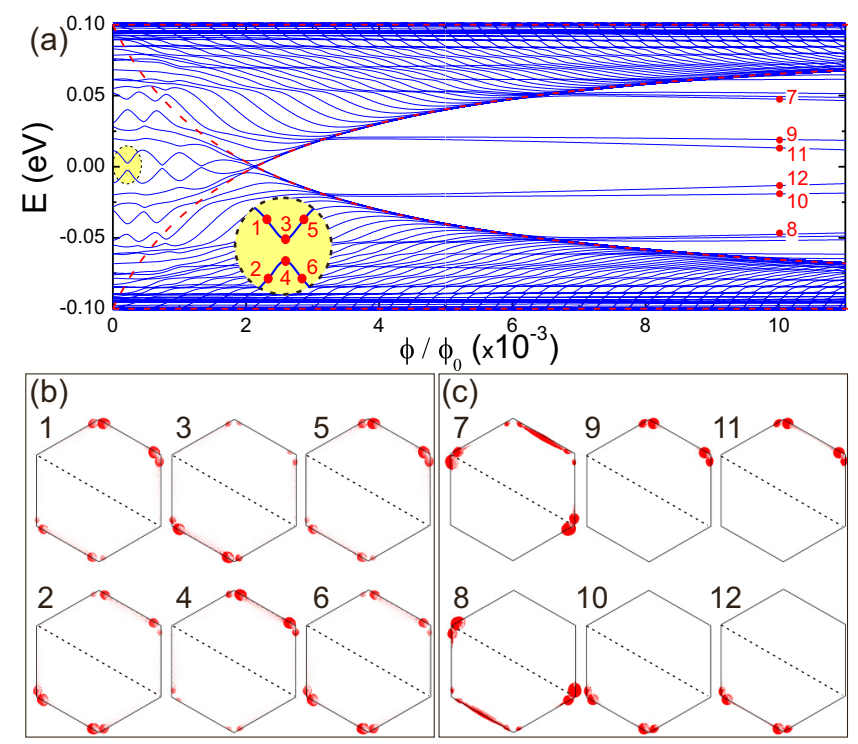

FIG. 9. (a) A zoom of the hexagonal zigzag QD energy spectrum [Fig. 8(b)] near the zero-energy level. The red dashed curves are the lowest zeroth LLs of an infinite sheet of BLG. (b),(c) The total probability densities are shown for states labeled by $1-2,3-4,5$ 6 , and $7-12$ at $\phi / \phi_{0}=0.15 \times 10^{-3}, 0.22 \times 10^{-3}, 0.3 \times 10^{-3}$, and $10.0 \times 10^{-3}$, respectively. Larger red circles represent higher-density amplitudes. 


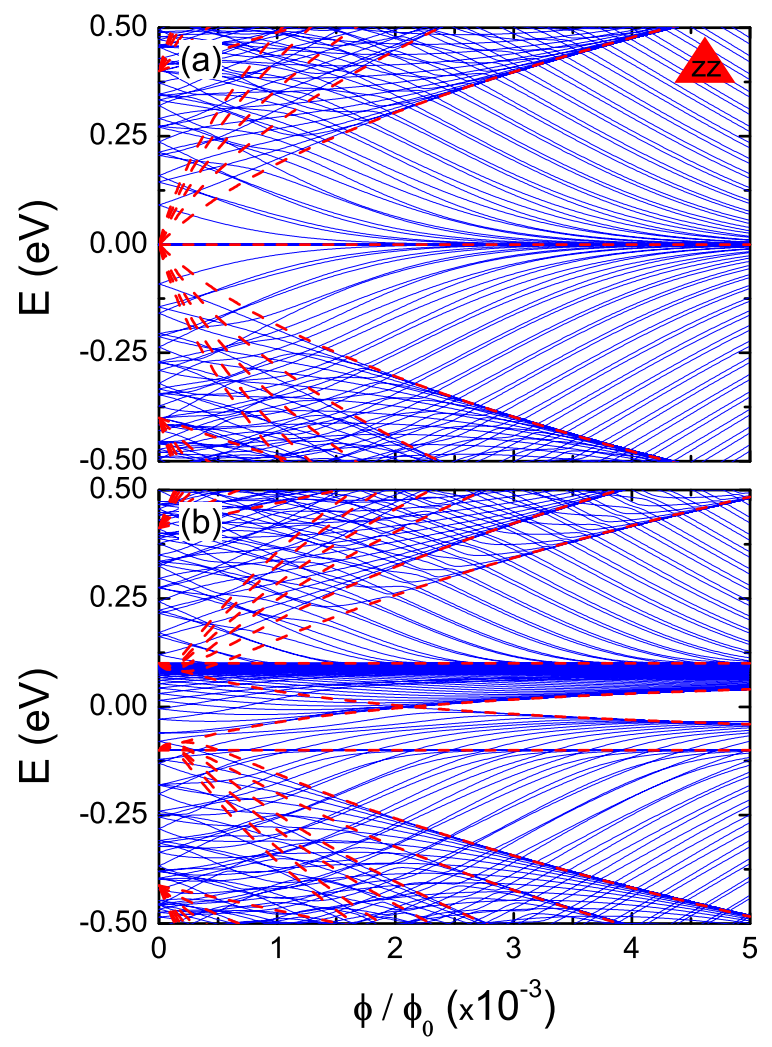

FIG. 10. The same as in Fig. 8, but for the zigzag triangular BLG QD with side length $L \approx 25.415 \mathrm{~nm}(N=103$ carbon hexagons along each side), as sketched in Fig. 1(d).

and hole levels. Our results demonstrate that these states are confined along the terminated-zigzag atoms with no interlayer links [see the structure of the zigzag hexagonal BLG QD in Fig. 1(c)]. Notice also that exactly at the anticrossing, states 3 and 4 are predominantly located at the corners of the hexagon that are composed of atoms from the top and bottom layers, respectively [see sketch in Fig. 1(c)], so that their energies must be slightly different, explaining the observed gap around $E=0$. The total probability densities show a reflection symmetry for electron and hole states along the black dashed lines in the panels of Figs. 9(b) and 9(c). Similar to the spectra of QDs with armchair edges (Figs. 6 and 7), we observe extra energy levels in the region $E_{+}^{(0)}<E<E_{-}^{(0)}$ and $\phi>\phi_{C}$. These states are labeled 7-8 in Fig. 9(a). The corresponding total densities are shown in Fig. 9(c). In contrast to the edge states in the region $\phi<\phi_{C}$, now the carriers are confined mostly around two corners of the dot, instead of the four as in Fig. 9(b). As one can see in Fig. 9(c), the states 9-10 and 11-12 exhibit confinement at some of the corners of the dot, whereas for the higher-energy states labeled 7-8, we see confinement both at the edges and the corners, as they approach the quantum Hall edge states, which are confined along the QD edges. The lowest states are grouped in pairs, i.e., 9-11 and $10-12$, that result from the symmetry of the wave functions [see corresponding probability densities in Fig. 9(c)].

Figures 10(a) and 10(b) show the energy spectrum as a function of the magnetic flux for a zigzag triangular BLG QD in the (a) absence and (b) presence of an applied gate potential,
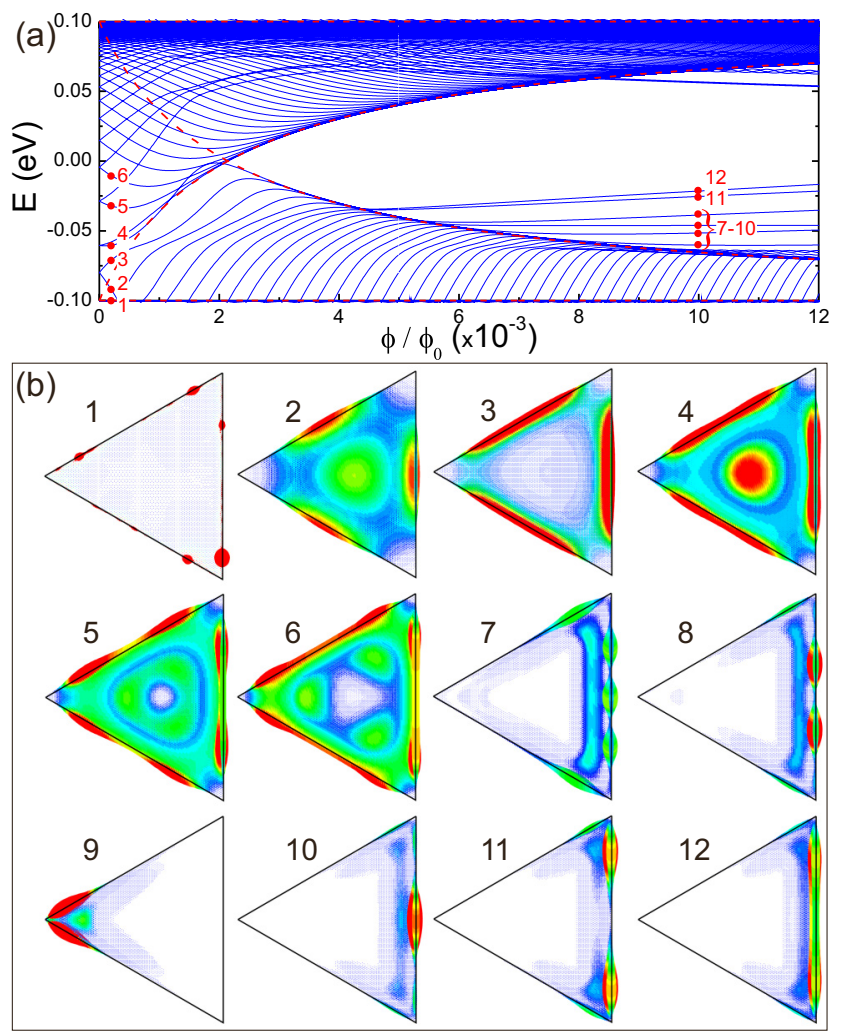

FIG. 11. (a) A zoom of the triangular zigzag magnetic energy spectrum [Fig. 10(b)] near the zero-energy level. The red dashed curves are the lowest zeroth LLs of an infinite sheet of BLG. (b) The total probability densities are shown for states labeled by the points 1 to 6 at $\phi / \phi_{0}=0.2 \times 10^{-3}$ and for points 7 to 12 at $\phi / \phi_{0}=10.0 \times 10^{-3}$. Red (blue) colors stand for high (low) density.

respectively. In contrast to hexagonal BLG QDs with zigzag edges (Fig. 8), the unbiased energy spectrum in Fig. 10(a) exhibits a $2(N-1)$ degenerate zero-energy state, i.e., $N-1$ zero states for each triangular layer, where $N$ is the number of hexagonal carbon rings along each side of the QD. As shown in Fig. 1(d), an $A B$-stacked triangular BLG QD with zigzag edges has a different edge structure in each layer, i.e., different number of edge atoms that are not linked to the other layer via the interlayer hopping [bottom (top) layer has 3 (1) nonlinked terminated edges]. This leads to the breaking of the layer symmetry, resulting in the breaking of electron-hole symmetry in the spectrum shown in Fig. 10(b) when $V \neq 0$. This corresponds to Fig. 7(b) of Ref. [34], where the spectrum in the absence of a magnetic field was shown to exhibit a clear electron-hole symmetry break.

The low-energy levels of a triangular BLG QD with zigzag edges are shown in Fig. 11(a). We plot the total electron density for the states labeled $1-6$ at $\phi / \phi_{0}=0.2 \times 10^{-3}$ and for those states labeled $7-10$ at $\phi / \phi_{0}=10.0 \times 10^{-3}$. The bias potential splits the $2(N-1)$ degenerate states into $N-1$ degenerate states with energy $E=-V$ that are confined at the edges of the lower layer with potential $-V$, while the other $N-1$ states spread into the region $E_{-}^{(0)} \leqslant E \leqslant V$. As the magnetic field increases, the twofold degeneracy of the levels is lifted, leading to crossings of these states. The total probability density for 
the $N$ th state with energy $E=-0.1 \mathrm{eV}$ is shown in panel 1 of Fig. 11(b), which confirms the strong confinement of the $N-1$ states at the edges of the lower layer. For higherenergy levels with $|E|<V$, the energy states (see panels 2-6) display a nodal character along both edges and inside the dot. This is reminiscent of quantum well states, where the number of nodes increases as we go to higher-energy levels. In contrast to the lowest states with $\phi<\phi_{C}$, the states in the region $\phi>\phi_{C}$ and $E_{+}^{(0)}<E<E_{-}^{(0)}$ exhibit confinement mostly at one edge of the triangular dot [see panels 7-8 and 1012 in Fig. 11(b)] . The total probability density of these states has a nodal character along that zigzag edge of the triangular QD which is not linked to the other layer [see Fig. 1(d)]. The state labeled 9 in Fig. 11(b) shows confinement at the corner of the dot.

\section{CIRCULAR BILAYER GRAPHENE QD: MIXTURE OF ARMCHAIR AND ZIGZAG EDGES}

It is interesting to investigate the energy spectrum of circular BLG QDs since this geometry has a combination of both zigzag and armchair edges at its boundary and is therefore relevant to the experimental samples with no uniform edges. We consider two kinds of circular BLG QDs: (i) a QD cut out from a BLG sheet [see Fig. 1(e)] and (ii) a circular BLG QD surrounded by a staggered potential media with $M_{0}=1.0 \mathrm{eV}$ [see Fig. 1(f)]. The staggered potential media can be realized by using an appropriate nanostructured substrate for BLG.

The energy levels for a circular BLG QD as a function of magnetic flux are shown in Fig. 12(a) in the absence of, and in Fig. 12(b) in the presence of, a bias potential for the case of a cutout circular QD with radius $R=10 \mathrm{~nm}$. Without bias potential, the energy spectrum is similar to that of a hexagonal BLG QD with zigzag edges [Fig. 8(a)], exhibiting a bunch of energy levels with $E \approx 0$. The spectrum in the presence of bias is, however, different from that of a zigzag hexagonal BLG QD [Fig. 8(b)]. Now the corner states [that appeared in the region $\phi>\phi_{C}$ and $E_{+}^{(0)}<E_{n}<E_{-}^{(0)}$ in Fig. 8(b)] are absent, which is due to the circular geometry having round boundaries [see Fig. 12(b)]. The formation of a subband, including six energy states, in the region $\phi<\phi_{C}$ and $E_{-}^{(0)}<E_{n}<E_{+}^{(0)}$ is related to the $C_{6}$ symmetry brought by the position of the zigzag edges in a circular BLG QD [see Fig. 1(e)]. This energy subband exhibits periodic oscillations as the magnetic field increases, which is reminiscent of the spectrum of hexagonal monolayer graphene quantum rings with zigzag edges under applied magnetic fields [45]. This is due to the fact that in the absence of field, the edge states have energies inside the gap generated by the bias. As the magnetic field increases, these states are pushed towards the center of the dot, while the gap prevents their wave functions from entering the dot, which is effectively the same situation that leads to AharonovBohm oscillations of energy states in quantum rings. Indeed, as one verifies in the inset of Fig. 12(b), the energy gap around $E=0$ is closed periodically as $\phi_{R}=(2 n+1) \phi_{0}$, with $n=$ $0,1,2, \ldots$, where $\phi_{R}=B \pi R^{2}$ is the magnetic flux threading the circular dot, as expected for Aharonov-Bohm oscillations in such a bilayer. Notice that, in fact, these oscillations are also present in the low-energy spectrum at $\phi<\phi_{C}$ for the

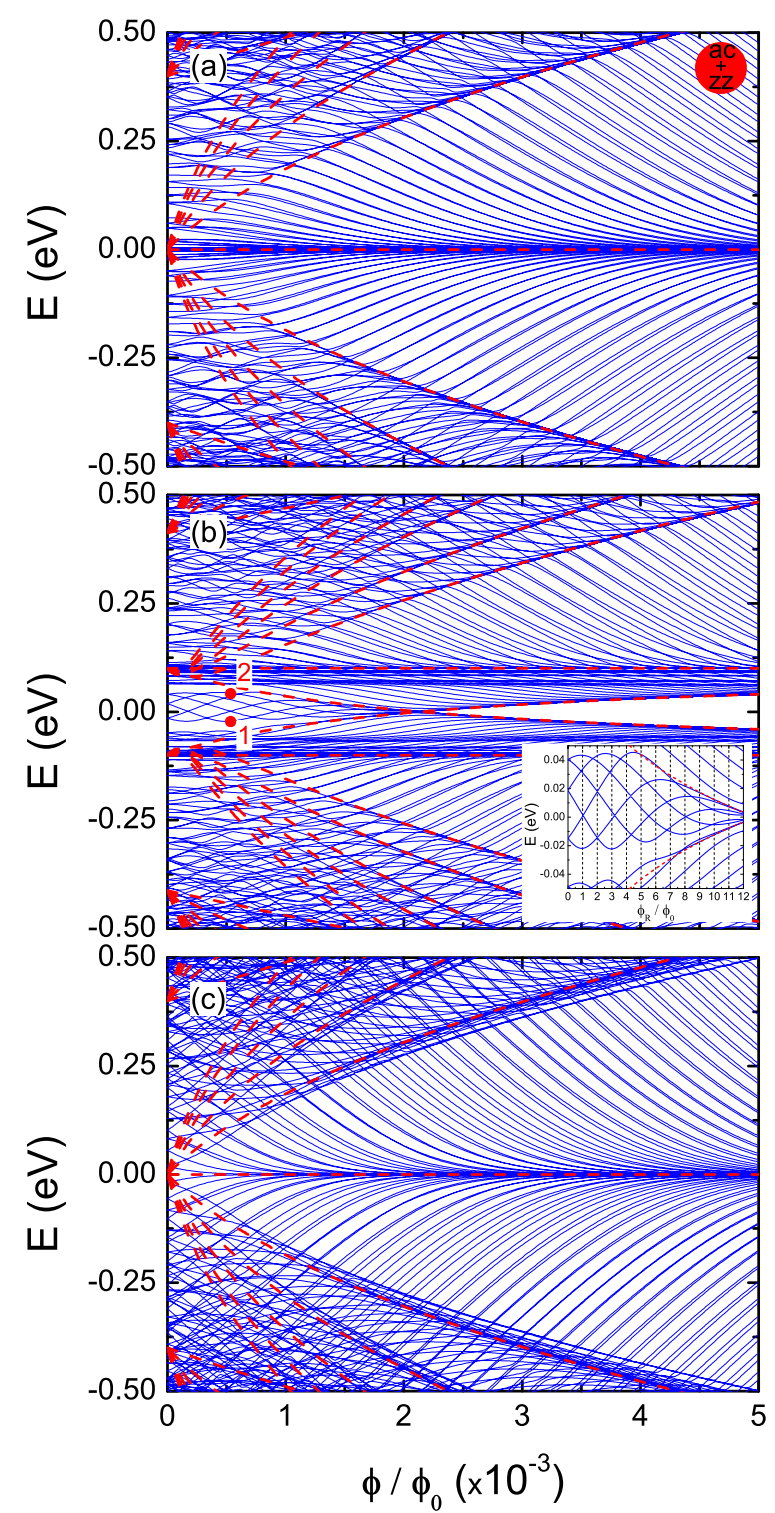

FIG. 12. Energy levels as a function of the magnetic flux $\left(\phi / \phi_{0}\right)$ for a circular BLG QD with radius $R=10 \mathrm{~nm}$ (a) in the absence and (b) in the presence of a bias potential, and (c) considering a mass potential profile surrounding an unbiased region, as sketched in Figs. 1(e) and 1(f), respectively. The red dashed curves are the LLs of an infinite sheet of BLG. The bias potential is $V=0.1 \mathrm{eV}$. The inset in (b) shows a zoom of the low-energy oscillations at low magnetic fields. $\phi_{R}=B \pi R^{2}$ is the magnetic flux threading the circular dot.

biased hexagonal zigzag QD [see Figs. 8(b) and 9(a)], where a similar situation of edge confinement is observed; but, in contrast to the circular case, gaps separating all oscillating energies are present that are a consequence the breaking of (i) the circular symmetry of the wave function, due to the hexagonal geometry, and (ii) the interlayer symmetry, due to the applied bias. In Fig. 13, we show the probability density corresponding to the energies labeled by points 1 and 2 in Fig. 12(b), which exhibit an electron-hole symmetry. It can be seen that the total density distribution for points 1 and 2 is rotated by $\pi / 3$. The densities are mostly confined at the six 


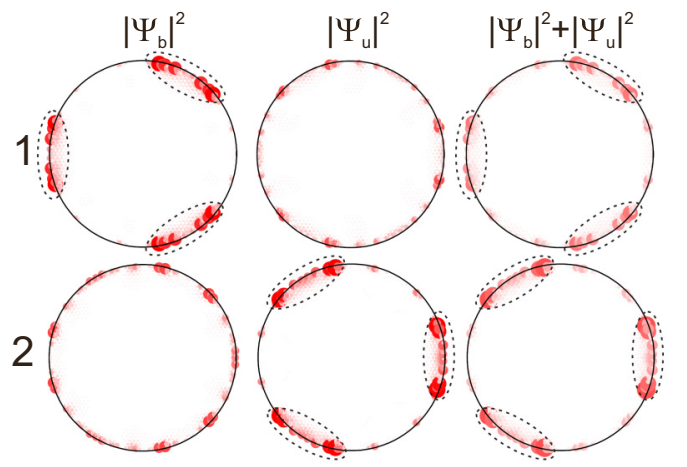

FIG. 13. The probability amplitude for the bottom $\left(\left|\Psi_{b}\right|^{2}\right)$ and top $\left(\left|\Psi_{u}\right|^{2}\right)$ layers, as well as the total contribution $\left(\left|\Psi_{b}\right|^{2}+\left|\Psi_{u}\right|^{2}\right)$ are shown for points 1 and 2 of the energy levels indicated in Fig. 12(b) at the magnetic flux $\left(\phi / \phi_{0}=0.5 \times 10^{-3}\right)$. The maximum amplitudes match with the six largest zigzag parts of the circular BLG QD with $R=10 \mathrm{~nm}$. Larger red circles represent higher-density amplitudes.

largest zigzag patches of the circular dot, and the total electron (hole) density is mostly composed of the contribution from the top (bottom) layer.

Let us now analyze the case of a circular BLG QD defined by a mass-term potential of $M_{0}=1.0 \mathrm{eV}$. Our purpose of considering such structure is to eliminate the influence of edges. The energy spectrum in this case is shown in Fig. 12(c) as a function of magnetic flux. It looks qualitatively similar to the one shown in Fig. 2(a), for a hexagonal BLG QD with armchair boundaries, where we have no zero-energy states due to the zigzag edges. However, qualitatively, the spectrum displays a smaller energy gap at low magnetic field as compared to the hexagonal case.

\section{CONCLUSIONS}

Using the tight-binding approach, we obtained the magnetic-field-dependent electron and hole energy spectra for hexagonal, triangular, and circular $A B$-stacked BLG QDs with zigzag and armchair edges. We investigated the magnetic field dependence of the energy levels both in the absence and in the presence of an applied electric field. Our results showed that the energy levels of BLG QDs depend strongly on the confinement geometry and that the different edge types play an important role. A comparison was made between the spectra for the different geometries and edges, with the circular BLG
QD defined by a mass-staggered potential. The latter was shown to be qualitatively similar to the results obtained for the hexagonal BLG QD with armchair edges, whereas the unbiased cutout circular QD spectrum looked qualitatively like the one for hexagonal BLG QD with zigzag edges.

All of the studied spectra obtained in this paper for both unbiased and biased BLG QDs preserve the electron $(e)$ hole $(h)$ symmetry, $E_{e}=-E_{h}$, except for the zigzag triangular BLG QD in the presence of an external gate potential. The asymmetry of this spectrum for $V \neq 0$ is a consequence of the different structure of the edge atoms in the upper and lower layers of the triangular BLG QDs, having a different number of interlayer nonconnected atoms. For unbiased BLG QDs, we found that the quantum Hall edge states approach the zeroth LL of an infinite BLG in pairs, which results from the layer symmetry in BLG QDs when $V=0$. We noticed that the BLG QDs with zigzag edges exhibit the well-known edge states, with a $2(N-1)$-fold-degenerate zero-energy level and a bunch of zero-energy states for the triangular and hexagonal QDs, respectively.

Due to the peculiar form of the LLs in biased BLG, our results in the presence of a bias potential showed different types of states that appear in different regions of the energy spectrum. We found that biased QDs exhibit states which are confined at the corners of the QD when the magnetic field is larger than $\phi_{C}$, with $\phi_{C}$ being the crossing point of the electron-hole zeroth LLs. These states, identified here as quantum Hall corner states, are shown to be absent for a circular geometry.

The unconventional energy states found inside the gap in biased BLG quantum dots can be probed, e.g., by attaching leads to the dot and measuring its transport properties, such as its magnetotransmission minima [46] and its chemical potential at the resonances in Coulomb diamond patterns [10,47]. In particular, quantum Hall corner states can also be probed by observing their local density of states in scanning tunneling microscopy (STM) experiments [48].

\section{ACKNOWLEDGMENTS}

This work was financially supported by $\mathrm{CNPq}$, under Contract No. NanoBioEstruturas 555183/2005-0, PRONEX/FUNCAP, CAPES Foundation under the Process No. BEX 7178/13-1, the Flemish Science Foundation (FWO$\mathrm{Vl}$ ), the Bilateral programme between $\mathrm{CNPq}$ and FWO-Vl, and the Brazilian Program Science Without Borders (CsF).
[1] A. Reina, S. Thiele, X. Jia, S. Bhaviripudi, M. Dresselhaus, J. Schaefer, and J. Kong, Nano Res. 2, 509 (2009)

[2] C. Soldano, A. Mahmood, and E. Dujardin, Carbon 48, 2127 (2010).

[3] G. Jo, M. Choe, S. Lee, W. Park, Y. H. Kahng, and T. Lee, Nanotechnology 23, 112001 (2012).

[4] K. S. Novoselov, A. K. Geim, S. V. Morozov, D. Jiang, Y. Zhang, S. V. Dubonos, I. V. Grigorieva, and A. A. Firsov, Science 306, 666 (2004).

[5] A. H. Castro Neto, F. Guinea, N. M. R. Peres, K. S. Novoselov, and A. K. Geim, Rev. Mod. Phys. 81, 109 (2009).
[6] J. Güttinger, T. Frey, C. Stampfer, T. Ihn, and K. Ensslin, Phys. Rev. Lett. 105, 116801 (2010).

[7] B. Trauzettel, D. V. Bulaev, D. Loss, and G. Burkard, Nat. Phys. 3, 192 (2007)

[8] P. G. Silvestrov and K. B. Efetov, Phys. Rev. Lett. 98, 016802 (2007).

[9] M. I. Katsnelson, K. S. Novoselov, and A. K. Geim, Nat. Phys. 2, 620 (2006).

[10] S. Schnez, F. Molitor, C. Stampfer, J. Güttinger, I. Shorubalko, T. Ihn, and K. Ensslin, Appl. Phys. Lett. 94, 012107 (2009). 
[11] D. A. Bahamon, A. L. C. Pereira, and P. A. Schulz, Phys. Rev. B 79, 125414 (2009).

[12] Z. Z. Zhang, K. Chang, and F. M. Peeters, Phys. Rev. B 77, 235411 (2008).

[13] M. Grujić, M. Zarenia, A. Chaves, M. Tadić, G. A. Farias, and F. M. Peeters, Phys. Rev. B 84, 205441 (2011).

[14] M. Zarenia, A. Chaves, G. A. Farias, and F. M. Peeters, Phys. Rev. B 84, 245403 (2011).

[15] F. Molitor, J. Güttinger, C. Stampfer, S. Dröscher, A. Jacobsen, T. Ihn, and K. Ensslin, J. Phys. Condens. Matter 23, 243201 (2011).

[16] H. P. Heiskanen, M. Manninen, and J. Akola, New J. Phys. 10, 103015 (2008).

[17] A. V. Rozhkov, G. Giavaras, Y. P. Bliokh, V. Freilikher, and F. Nori, Phys. Rep. 503, 77 (2011).

[18] M. Calvo, Phys. Rev. B 84, 235413 (2011).

[19] J. H. Bardarson, M. Titov, and P. W. Brouwer, Phys. Rev. Lett. 102, 226803 (2009).

[20] J. Park, S. B. Jo, Y.-J. Yu, Y. Kim, J. W. Yang, W. H. Lee, H. H. Kim, B. H. Hong, P. Kim, K. Cho, and K. S. Kim, Adv. Mater. 24, 407 (2012).

[21] S. Dröscher, J. Güttinger, T. Mathis, B. Batlogg, T. Ihn, and K. Ensslin, Appl. Phys. Lett. 101, 043107 (2012).

[22] E. V. Castro, K. S. Novoselov, S. V. Morozov, N. M. R. Peres, J. M. B. Lopes dos Santos, J. Nilsson, F. Guinea, A. K. Geim, and A. H. Castro Neto, Phys. Rev. Lett. 99, 216802 (2007).

[23] F. Xia, D. B. Farmer, Y. M. Lin, and P. Avouris, Nano Lett. 10, 715 (2010).

[24] M. T. Allen, J. Martin, and A. Yacoby, Nat. Commun. 3, 934 (2012).

[25] A. M. Goossens, S. C. M. Driessen, T. A. Baart, K. Watanabe, T. Taniguchi, and L. M. K. Vandersypen, Nano Lett. 12, 4656 (2012).

[26] E. McCann, Phys. Rev. B 74, 161403(R) (2006).

[27] J. M. Pereira, Jr., P. Vasilopoulos, and F. M. Peeters, Nano Lett. 7, 946 (2007).

[28] J. M. Pereira, Jr., F. M. Peeters, and P. Vasilopoulos, Phys. Rev. B 76, 115419 (2007).

[29] A. Matulis and F. M. Peeters, Phys. Rev. B 77, 115423 (2008).

[30] J. M. Pereira, Jr., F. M. Peeters, P. Vasilopoulos, R. N. Costa Filho, and G. A. Farias, Phys. Rev. B 79, 195403 (2009).
[31] P. Recher, J. Nilsson, G. Burkard, and B. Trauzettel, Phys. Rev. B 79, 085407 (2009).

[32] D. P. Zebrowski, E. Wach, and B. Szafran, Phys. Rev. B 88, 165405 (2013).

[33] D. R. da Costa, M. Zarenia, Andrey Chaves, G. A. Farias, and F. M. Peeters, Carbon 78, 392 (2014).

[34] D. R. da Costa, M. Zarenia, Andrey Chaves, G. A. Farias, and F. M. Peeters, Phys. Rev. B 92, 115437 (2015).

[35] Y. B. Zhang, T. T. Tang, C. Girit, Z. Hao, M. C. Martin, A. Zettl, M. F. Crommie, Y. R. Shen, and F. Wang, Nature (London) 459, 820 (2009).

[36] D. Wang and G. Jin, Europhys. Lett. 92, 57008 (2010).

[37] G. Giavaras, P. A. Maksym, and M. Roy, J. Phys. Condens. Matter 21, 102201 (2009).

[38] Y. P. Bliokh, V. Freilikher, and F. Nori, Phys. Rev. B 81, 075410 (2010).

[39] T. Champel and S. Florens, Phys. Rev. B 82, 045421 (2010).

[40] G. Giavaras and F. Nori, Phys. Rev. B 85, 165446 (2012).

[41] E. McCann and M. Koshino, Rep. Prog. Phys. 76, 056503 (2013).

[42] S. Y. Zhou, G.-H. Gweon, A. V. Fedorov, P. N. First, W. A. de Heer, D.-H. Lee, F. Guinea, A. H. Castro Neto, and A. Lanzara, Nat. Mater. 6, 770 (2007).

[43] G. Giovannetti, P. A. Khomyakov, G. Brocks, P. J. Kelly, and J. van den Brink, Phys. Rev. B 76, 073103 (2007)

[44] M. S. Nevius, M. Conrad, F. Wang, A. Celis, M. N. Nair, A. Taleb-Ibrahimi, A. Tejeda, and E. H. Conrad, Phys. Rev. Lett. 115, 136802 (2015).

[45] D. R. da Costa, A. Chaves, M. Zarenia, J. M. Pereira, Jr., G. A. Farias, and F. M. Peeters, Phys. Rev. B 89, 075418 (2014).

[46] S. Maëro, A. Torche, T. Phuphachong, E. Pallecchi, A. Ouerghi, R. Ferreira, L.-A. de Vaulchier, and Y. Guldner, Phys. Rev. B 90, 195433 (2014)

[47] J. Güttinger, C. Stampfer, F. Libisch, T. Frey, J. Burgdörfer, T. Ihn, and K. Ensslin, Phys. Rev. Lett. 103, 046810 (2009).

[48] D. Subramaniam, F. Libisch, Y. Li, C. Pauly, V. Geringer, R. Reiter, T. Mashoff, M. Liebmann, J. Burgdörfer, C. Busse, T. Michely, R. Mazzarello, M. Pratzer, and M. Morgenstern, Phys. Rev. Lett. 108, 046801 (2012). 